\title{
Neutrino Mass Matrix Related to Up-Quark Masses and Nearly Tribimaximal Mixing - Based on a Yukawaon model -1
}

\author{
Yoshio Koide \\ IHERP, Osaka University, Toyonaka, Osaka 560-0043, Japan \\ E-mail: koide@het.phys.sci.osaka-u.ac.jp
}

\begin{abstract}
Based on a new approach (the so-called Yukawaon model) to the mass spectra and mixings, a neutrino mass matrix which is described in terms of the up-quark masses and CKM matrix parameters is proposed. The mass matrix successfully leads to a nearly tribimaximal mixing without assuming any discrete symmetry.
\end{abstract}

\section{What is a Yukawaon model?}

In the standard model of the quarks and leptons, we have many parameters in the theory. Especially, since the Yukawa coupling constants $Y_{f}(f=u, d, e, \nu)$ are entirely free, we have no predictions for the mass spectra and mixings. Therefore, usually, we assume a flavor symmetry, and thereby, we discussed relations among the mass spectra and mixings. However, if we want to bring a flavor symmetry into the standard model, we encounter a no-go theorem [1, 2] on the flavor symmetry, so that we cannot impose any flavor symmetry on the standard model. Of course, we can evade 2] the no-go theorem if we consider a multi-Higgs model in which Higgs scalars have flavor quantum numbers. However, such multi-Higgs models will newly bring some troubles, e.g. flavor-changing neutral current problem, rapid evolutions of coupling constants, and so on.

There is another idea for the origin of the mass spectra and mixings: In the Yukawa interactions

$$
H_{Y}=\sum_{i, j} \bar{q}_{L}^{i}\left(Y_{u}\right)_{i}^{j} u_{R j} H_{u}+\cdots
$$

we regard the Yukawa coupling constants $Y_{f}$ as "effective" coupling constants $Y_{f}^{\text {eff }}$ in an effective theory, and we consider that $Y_{f}^{e f f}$ originate in vacuum expectation values (VEVs) of new scalars $Y_{f}$, i.e.

$$
Y_{f}^{e f f}=\frac{y_{f}}{\Lambda}\left\langle Y_{f}\right\rangle
$$

where $\Lambda$ is a scale of the effective theory, i.e.

$$
H_{Y}=\sum_{i, j} \frac{y_{u}}{\Lambda} \bar{q}_{L}^{i}\left(Y_{u}\right)_{i}^{j} u_{R j} H_{u}+\cdots
$$

\footnotetext{
1 A talk given at "Particle Physics, Astrophysics and Quantum Field Theory: 75 Years since Solvay" (PAQFT08), 27-29 Nov. 2008, Nanyang Executive Centre, Singapore. To appear in the Conference Proceedings (Intl. J. Mod. Phys. A).
} 
We refer the fields $Y_{f}$ as "Yukawaons" 3] hereafter. Note that in the Yukawaon model, the Higgs scalars are the same as ones in the conventional model, i.e. we consider only two Higgs scalars $H_{u}$ and $H_{d}$ as the origin of the masses (not as the origin of the mass spectra). It should be noted that the Yukawaons $Y_{f}$ are gauge singlets.

The VEVs of Yukawaons are obtained from supersymmetric (SUSY) vacuum conditions for a superpotential $W$. In the conventional approach, the masses and mixings are calculated by diagonalizing the mass matrices $M_{f}$ which are constrained by flavor symmetries, while, in the Yukawaon approach, those are obtained by writing a superpotential under flavor symmetries and by solving simultaneous equations from the SUSY vacuum conditions. For example, we assume an $\mathrm{O}(3)$ flavor symmetry [4] and we consider that the Yukawaons $Y_{f}$ are $(\mathbf{3} \times \mathbf{3})_{S}=\mathbf{1}+\mathbf{5}$ of $\mathrm{O}(3)_{F}$ :

$$
\begin{gathered}
W_{Y}=\sum_{i, j} \frac{y_{u}}{\Lambda} u_{i}^{c}\left(Y_{u}\right)_{i j} q_{j} H_{u}+\sum_{i, j} \frac{y_{d}}{\Lambda} d_{i}^{c}\left(Y_{d}\right)_{i j} q_{j} H_{d} \\
+\sum_{i, j} \frac{y_{\nu}}{\Lambda} \ell_{i}\left(Y_{\nu}\right)_{i j} \nu_{j}^{c} H_{u}+\sum_{i, j} \frac{y_{e}}{\Lambda} \ell_{i}\left(Y_{e}\right)_{i j} e_{j}^{c} H_{d}+\text { h.c. }+\sum_{i, j} y_{R} \nu_{i}^{c}\left(Y_{R}\right)_{i j} \nu_{j}^{c},
\end{gathered}
$$

where $q$ and $\ell$ are $\mathrm{SU}(2)_{L}$ doublet fields, and $f^{c}(f=u, d, e, \nu)$ are $\mathrm{SU}(2)_{L}$ singlet fields. Here, in order to distinguish each $Y_{f}$ from others, we have assigned $\mathrm{U}(1)_{X}$ charges as $Q_{X}\left(f^{c}\right)=-x_{f}$, $Q_{X}\left(Y_{f}\right)=+x_{f}$ and $Q_{X}\left(Y_{R}\right)=2 x_{\nu}$. We also write superpotential terms $W_{f}$ which are introduced in order to fix the VEVs of $Y_{f}$ under the $\mathrm{O}(3)$ flavor symmetry and $\mathrm{U}(1)_{X}$ symmetry, and we obtain simultaneous equations for the $\operatorname{VEVs}\left\langle Y_{f}\right\rangle$ by calculating SUSY vacuum conditions for $W=W_{Y}+W_{u}+W_{d}+\cdots$.

In the next section, we give a short review of a mass spectrum of the charged leptons as an example of the supersymmetric Yukawaon approach. We will discuss ratios

$$
R_{e} \equiv \frac{m_{e}+m_{\mu}+m_{\tau}}{\left(\sqrt{m_{e}}+\sqrt{m_{\mu}}+\sqrt{m_{\tau}}\right)^{2}}
$$

and

$$
r_{e} \equiv \frac{\sqrt{m_{e} m_{\mu} m_{\tau}}}{\left(\sqrt{m_{e}}+\sqrt{m_{\mu}}+\sqrt{m_{\tau}}\right)^{3}}
$$

In Sec.3, we propose a curious neutrino mass matrix 2

$$
M_{\nu}=k_{\nu} M_{e}\left(M_{e} M_{u}^{1 / 2}+M_{u}^{1 / 2} M_{e}\right)^{-1} M_{e}
$$

which is related to up-quark mass matrix $M_{u}$, and which can leads to a nearly tribimaximal mixing without assuming any discrete symmetry. (The tribimaximal mixing has usually been explained by assuming a discrete symmetry for the lepton mass matrices. [5]) Finally, Sec.4 is devoted to summary and concluding remarks.

\footnotetext{
${ }^{2}$ The form (1.7) has already been proposed in Ref. 3. Although five Yukawaons $Y_{u}, Y_{d}, Y_{e}, Y_{\nu}$ and $Y_{R}$ have been assumed in Ref. 3, in the present scenario, we will assume only four Yukawaons $Y_{u}, Y_{d}, Y_{e}$ and $Y_{R}$.
} 


\section{Mass spectrum}

In order to see how to evaluate the mass spectra in the Yukawaon model, let us show an example in the charged lepton sector.

Under the $\mathrm{O}(3)$ flavor symmetry and $\mathrm{U}(1)_{X}$ symmetry, we can write the following superpotential terms 6 ]

$$
\begin{gathered}
W_{A}=\lambda_{A}\left[\Phi_{e} \Phi_{e} A_{e}\right]+\mu_{A}\left[Y_{e} A_{e}\right]+\lambda_{A}^{\prime}\left[\Phi_{e} \Phi_{e} A_{e}^{\prime}\right]+\mu_{A}^{\prime}\left[Y_{e} A_{e}^{\prime}\right] \\
+\lambda_{A}^{\prime \prime}\left[\hat{\Phi}_{e} \hat{\Phi}_{e} A_{e}^{\prime}\right]+\mu_{A}^{\prime \prime}\left[Y_{e}^{\prime} A_{e}^{\prime}\right]
\end{gathered}
$$

where, for simplicity, we have denoted $\operatorname{Tr}[X]$ as $[X]$ concisely. Here, the field $\Phi_{e}$ has been introduced in order to fix the VEV values of $Y_{e}$. We will refer $\Phi_{e}$ as an "ur-Yukawaon". The field $\hat{\Phi}_{e}$ denotes a traceless part of the ur-Yukawaon $\Phi_{e}$, i.e. $\hat{\Phi}_{e}=\Phi_{e}-\frac{1}{3}\left[\Phi_{e}\right]$, and we have assigned $\mathrm{U}(1)_{X}$ charges as $Q_{X}\left(Y_{e}\right)=x_{e}, Q_{X}\left(\Phi_{e}\right)=\frac{1}{2} x_{e}$ and $Q_{X}\left(A_{e}\right)=Q_{X}\left(A_{e}^{\prime}\right)=-x_{e}$. (In order to prevent $\left(Y_{e}^{\prime}\right)_{i j}$ from coupling with $\ell_{i} e_{j}^{c}$, we have to assign a different $\mathrm{U}(1)_{X}$ charge to the field $Y_{e}^{\prime}$ from the Yukawaon $Y_{e}$, so that we replace [6] the coefficient $\mu_{A}^{\prime \prime}$ with $\lambda_{A}^{\prime \prime \prime} \phi_{x}$, where $Q_{X}\left(\phi_{x}\right)=x_{\phi}$ and $Q_{X}\left(Y_{e}^{\prime}\right)=x_{e}-x_{\phi}$. However, for simplicity, hereafter, we use the expression $\mu_{A}^{\prime \prime}$ with $\mathrm{U}(1)_{X}$ charge $x_{\phi}$ instead of $\lambda_{A}^{\prime \prime \prime} \phi_{x}$. ) In Eq.(2.1), we have assumed non-existence of a term $\left[Y_{e}^{\prime} A_{e}\right]$. This is an ad hoc assumption, but it is a crucial assumption to obtain a charged lepton mass relation (2.10) later.

From the SUSY vacuum condition $\partial W / \partial A_{e}=0$, and $\partial W / \partial A_{e}^{\prime}=0$, we obtain the VEV relations

$$
Y_{e}=k \Phi_{e} \Phi_{e}
$$

and

$$
Y_{e}^{\prime}=k^{\prime}\left(\Phi_{e} \Phi_{e}+\xi \hat{\Phi}_{e} \hat{\Phi}_{e}\right)
$$

respectively, where $k=-\lambda_{A} / \mu_{A}$ and

$$
k^{\prime}=-\frac{1}{\mu_{A}^{\prime \prime}}\left(\lambda_{A}^{\prime}-\frac{\mu_{A}^{\prime}}{\mu_{A}} \lambda_{A}\right), \quad \xi=\frac{\lambda_{A}^{\prime \prime}}{\lambda_{A}^{\prime}-\frac{\mu_{A}^{\prime}}{\mu_{A}} \lambda_{A}} .
$$

Here, in Eq.(2.3), we have used the relation (2.2).

Next, we introduce a field $B_{e}$ with $Q_{X}=-\frac{3}{2} x_{e}+x_{\phi}$, and we write a superpotential term

$$
W_{B}=\lambda_{B}\left[\Phi_{e} Y_{e}^{\prime} B_{e}\right]+\varepsilon_{1} \lambda_{B}\left[\Phi_{e}\right]\left[Y_{e}^{\prime}\right]\left[B_{e}\right]
$$

(For a more general form, see Ref.[6].) The SUSY vacuum condition $\partial W / \partial B_{e}=0\left(W=W_{e}=\right.$ $\left.W_{A}+W_{B}\right)$ gives

$$
\begin{gathered}
\Phi_{e}\left(\Phi_{e} \Phi_{e}+\xi \hat{\Phi}_{e} \hat{\Phi}_{e}\right)+\varepsilon_{1}\left[\Phi_{e}\right]\left[\Phi_{e} \Phi_{e}+\xi \hat{\Phi}_{e} \hat{\Phi}_{e}\right] \mathbf{1} \\
=(1+\xi) \Phi_{e}^{3}-\frac{2}{3} \xi\left[\Phi_{e}\right] \Phi_{e}^{2}+\frac{1}{9} \xi\left[\Phi_{e}\right]^{2} \Phi_{e} \\
+\varepsilon_{1}\left[\Phi_{e}\right]\left((1+\xi)\left[\Phi_{e} \Phi_{e}\right]-\frac{\xi}{3}\left[\Phi_{e}\right]^{2}\right) \mathbf{1}=0
\end{gathered}
$$


from Eq.(2.3). (Other SUSY vacuum conditions $\partial W / \partial Y_{e}=0$ and $\partial W / \partial \Phi_{e}=0$ lead to $A_{e}=$ $B_{e}=0$.) On the other hand, in general, in a cubic equation

$$
\Phi^{3}+c_{2} \Phi^{2}+c_{1} \Phi+c_{0} \mathbf{1}=0
$$

the coefficients $c_{i}$ have the following relations:

$$
c_{2}=-[\Phi], \quad c_{1}=\frac{1}{2}\left([\Phi]^{2}-[\Phi \Phi]\right), \quad c_{0}=-\operatorname{det} \Phi .
$$

In order to get non-zero a solution $\left[\Phi_{e}\right] \neq 0$, we must take

$$
\xi=-3
$$

from the coefficient $c_{2}$. Then, we can obtain the ratios

$$
R_{e} \equiv \frac{m_{e}+m_{\mu}+m_{\tau}}{\left(\sqrt{m_{e}}+\sqrt{m_{\mu}}+\sqrt{m_{\tau}}\right)^{2}}=\frac{\left[\Phi_{e} \Phi_{e}\right]}{\left[\Phi_{e}\right]^{2}}=1-\frac{2 \xi}{9(1+\xi)}=\frac{2}{3}
$$

and

$$
r_{e}=\frac{\sqrt{m_{e} m_{\mu} m_{\tau}}}{\left(\sqrt{m_{e}}+\sqrt{m_{\mu}}+\sqrt{m_{\tau}}\right)^{3}}=\frac{\operatorname{det} \Phi_{e}}{\left[\Phi_{e}\right]^{3}}=-\frac{1}{6} \varepsilon_{1},
$$

from the coefficients $c_{1}$ and $c_{0}$, respectively. Thus, we can obtain the successful relation [7] (2.10) for the charged lepton masses. At present, the parameter $\varepsilon_{1}$ is free, so that we cannot predict the value of $r_{e}$.

By the way, since we have successfully obtained the relation (2.10) without any adjustable parameter, another problem has risen in the present scenario: We know that $R=2 / 3$ is valid only for the charged lepton masses, and the observed masses for another sectors do not satisfy $R=2 / 3$. For example, the ratio $R_{u}$ for the up-quark masses is $R_{u} \simeq 8 / 9$ [8]. Can we modify the present scenario as it leads to $R_{u} \simeq 8 / 9$ ? At present it seems to be impossible, because there is no adjustable parameter in the present scenario. We expect that all Yukawaons are related to each other and those VEVs are described in terms of the VEV of the ur-Yukawaon $\Phi_{e}$. (A possibility that $\left\langle Y_{u}\right\rangle$ is described in terms of $\left\langle Y_{e}\right\rangle$ is discussed in Ref. [6] ). However, the purpose of the present talk is not to give the mass spectra, we do not discuss more details of this topic here. Our goal is to give a unified description of all Yukawaons. As the first step, we discuss a phenomenological relation between the Yukawaons $Y_{e}$ and $Y_{u}$ in the next section.

\section{$3 \quad$ Neutrino mass matrix}

Based on a supersymmetric Yukawaon model, a curious neutrino mass matrix has recently been proposed. 4. The mass matrix $M_{\nu}$ is related to up-quark masses as follows:

$$
M_{\nu}=M_{D} M_{R}^{-1} M_{D}^{T}
$$


where the neutrino Dirac mass matrix $M_{D}$ is given by

$$
M_{D} \propto\left\langle Y_{\nu}\right\rangle \propto\left\langle Y_{e}\right\rangle \propto M_{e}
$$

and the right-handed neutrino Majorana mass matrix $M_{R}$ is given by

$$
M_{R} \propto\left\langle Y_{R}\right\rangle \propto\left\langle Y_{e}\right\rangle\left\langle\Phi_{u}\right\rangle+\left\langle\Phi_{u}\right\rangle\left\langle Y_{e}\right\rangle \propto M_{e} M_{u}^{1 / 2}+M_{u}^{1 / 2} M_{e}
$$

where the ur-Yukawaon $\Phi_{u}$ has a relation $Y_{u}=k_{u} \Phi_{u} \Phi_{u}$ similar to Eq.(2.2).

In the present model, differently from the model given in Ref. [4], we assume that $\nu^{c}$ and $e^{c}$ have the same $\mathrm{U}(1)_{X}$ charge $x_{e}$, so that the Yukawaon $Y_{e}$ couples not only to the charged lepton sector, but also to the neutrino sector:

$$
W_{Y}=\frac{y_{\nu}}{\Lambda}\left(\ell Y_{e} \nu^{c}\right) H_{u}+\frac{y_{e}}{\Lambda}\left(\ell Y_{e} e^{c}\right) H_{d}+y_{R}\left(\nu^{c} Y_{R} \nu^{c}\right)+\frac{y_{R}^{\prime}}{\Lambda}\left(\nu^{c} Y_{e} Y_{e} \nu^{c}\right)
$$

Next, we assume additional fields $A_{R}$ with $Q_{X}=-\left(\frac{1}{2} x_{u}+x_{e}\right)$, so that we obtain the superpotential terms for $A_{R}$ as follows 3

$$
W_{R}=\lambda_{R}\left[\left(Y_{e} \Phi_{u}+\Phi_{u} Y_{e}\right) A_{R}\right]+\mu_{R}\left[Y_{R} A_{R}\right]
$$

Then, from the SUSY vacuum condition $\partial W / \partial A_{R}=0$, we can obtain the relation (3.3).

In order to calculate lepton mixing matrix $U$, we have to know a matrix form $M_{\nu}$ at the diagonal basis of $\left\langle Y_{e}\right\rangle$. Hereafter, we will denote a VEV matrix of a field $A$ at the diagonal basis of $\left\langle Y_{f}\right\rangle$ as $\langle A\rangle_{f}$. From the definition of the diagonal basis, we can express

$$
\begin{gathered}
\left\langle Y_{e}\right\rangle_{e} \propto D_{e}=\operatorname{diag}\left(m_{e}, m_{\mu}, m_{\tau}\right), \\
\left\langle\Phi_{u}\right\rangle_{e} \neq\left\langle\Phi_{u}\right\rangle_{u} \propto D_{u}^{1 / 2}=\operatorname{diag}\left(\sqrt{m_{u}}, \sqrt{m_{c}}, \sqrt{m_{t}}\right) .
\end{gathered}
$$

Our concern is in the form of $\left\langle\Phi_{u}\right\rangle_{e}$. The Cabibbo-Kobayashi-Maskawa (CKM) matrix $V$ satisfies the following relation 4

$$
\left\langle Y_{u}\right\rangle_{d}=V^{T}\left(\delta_{q}\right)\left\langle Y_{u}\right\rangle_{u} V\left(\delta_{q}\right) .
$$

On the analogy of the form of $\left\langle Y_{u}\right\rangle_{d},(3.8)$, we assume a form of $\left\langle\Phi_{u}\right\rangle_{e}$ as

$$
\left\langle\Phi_{u}\right\rangle_{e}=V^{T}\left(\delta_{\ell}\right)\left\langle\Phi_{u}\right\rangle_{u} V\left(\delta_{\ell}\right),
$$

where we have assumed that $\delta_{\ell}$ is a free parameter. Then, from observed values of the upquark masses [9] and CKM parameters[10], we obtain numerical results of the neutrino mixing parameters $\sin ^{2} 2 \theta_{23}, \tan ^{2} \theta_{12}$ and $\left|U_{13}\right|$ as shown in Table 1.

The observed value [10] of $\delta_{q}$ in the quark sector is $\delta_{q}=68.9^{\circ}$, but the value $\delta_{\ell}=\delta_{q}=68.9^{\circ}$ can not give a reasonable value of $\sin ^{2} 2 \theta_{23}$. On the other hand, the value $\delta_{\ell}=180^{\circ}$ can

\footnotetext{
${ }^{3}$ Exactly speaking, we have to read $\mu_{R}$ as $\lambda_{R}^{\prime} \phi_{R}$. Otherwise, the field $Y_{R}$ has the $\mathrm{U}(1)_{X}$ charge $Q_{X}\left(Y_{R}\right)=$ $2 x_{e}=\frac{1}{2} x_{u}+x_{e}$, so that we are obliged to accept the relation $x_{e}=\frac{1}{2} x_{u}$. This means that $Y_{e}$ and $\Phi_{u}$ have the same charge, a mixing between $Y_{e}$ and $\Phi_{u}$ is caused.

${ }^{4}$ In the present $\mathrm{O}(3)$ flavor model, the mass matrix $M_{f}$ is diagonalized as $U_{f}^{T} M_{f} U_{f}=D_{f}$.
} 
Table 1. Numerical results for neutrino mixing parameters versus $\delta_{\ell}$.

\begin{tabular}{cllll}
\hline$\delta_{\ell}$ & $\sin ^{2} 2 \theta_{23}$ & $\tan ^{2} \theta_{12}$ & $\left|U_{13}\right|$ & $\Delta m_{21}^{2} / \Delta m_{32}^{2}$ \\
\hline 0 & 0.4803 & 0.4745 & 0.01042 & 0.00196 \\
$60^{\circ}$ & 0.7631 & 0.4801 & 0.00844 & 0.00139 \\
$68.9^{\circ}$ & 0.8127 & 0.4851 & 0.00781 & 0.00127 \\
$90^{\circ}$ & 0.9028 & 0.5017 & 0.00615 & 0.00102 \\
$120^{\circ}$ & 0.9688 & 0.5277 & 0.00386 & 0.00081 \\
$180^{\circ}$ & 0.9952 & 0.5525 & 0.00094 & 0.00068 \\
\hline
\end{tabular}

successfully give a nearly tribimaximal mixing. Since, in the present $\mathrm{O}(3)$ model, the parameter $\delta_{\ell}$ must be 0 or $\pi$ because we have assumed that $\left\langle\Phi_{e}\right\rangle$ and $\left\langle\Phi_{u}\right\rangle$ are real. This is in favor of the results in Table 1. Although the values of $\Delta m_{21}^{2} / \Delta m_{32}^{2}$ in Table 1 are too small compared with the observed value[12, 11] $\Delta m_{21}^{2} / \Delta m_{32}^{2}=0.028 \pm 0.004$, the values can suitably be adjusted by considering the $y_{R}^{\prime}$-term in Eq.(3.4) without changing the predictions of the neutrino mixing parameters.

\section{Concluding remarks}

In conclusion, we have obtained a curious neutrino mass matrix (1.7) which is related to upquark mass matrix, and which can leads to a nearly tribimaximal mixing without assuming any discrete symmetry. (Although the form (1.7) has already been given in Ref. [4, the superpotential form in the present Yukawaon model is somewhat different from that in Ref. 44.) However, at present, there is no theoretical ground for the relation (3.9). The relation (3.9) is merely a phenomenological assumption. Nevertheless, we have successfully obtained a nearly tribimaximal mixing under this assumption without assuming any discrete symmetry. It will offer an important clue to a unified understanding of quarks and leptons to investigate why the phenomenological assumption (3.9) is so effectual.

The approach based on a Yukawaon model to masses and mixings will provide a new view different from conventional mass matrix models. Especially, the approach seems to be powerful to the predictions of mass relations $R_{f}$ and $r_{f}$ similar to $R_{e}$ and $r_{e}$ defined in Eqs.(1.5) and (1.6), respectively.

The neutrino mass matrix (1.7) can successfully give a tribimaximal mixing without assuming any discrete symmetry. Here, the neutrino mass matrix has been described by VEVs of the ur-Yukawaons $\Phi_{e}$ and $\Phi_{u}$. This suggests a possible relation between Yukawaons in the lepton and quark sectors. Our goal is to give a unified description of all Yukawaons. There is a possibility that VEVs of all Yukawaons $Y_{f}$ are described only one VEV matrix of the ur-Yukawaon $Y_{e}$. So far, we did not discuss the down-quark Yukawaon $Y_{d}$. Whether a unified Yukawaon model is possible or not is dependent on whether a $Y_{d}$ can also reasonably be described in terms of $\Phi_{e}$ (and also $\Phi_{u}$ ). This will be a touchstone of the Yukawaon approach.

At present, the calculations have been done in the supersymmetric limit. We have considered $\left\langle\Phi_{f}\right\rangle \sim \Lambda \sim 10^{15} \mathrm{GeV}$, so that almost Yukawaon components are massive and invisible in 
the low energy phenomena. However, some of Yukawaons are massless in the supersymmetric

limit. Since we consider an explicitly broken term of the flavor symmetry which effectively appears when SUSY is broken, those massless particles will acquire masses of $\mathrm{TeV}$ scale, so that the effects will be able to be observed in the $\mathrm{TeV}$ region physics.

\section{References}

[1] Y. Koide, Phys. Rev. D 71, 016010 (2005).

[2] Y. Koide, Invited talk presented at GUT 2007, Kusatsu, Japan, Dec. 17 - 19, 2007, in AIP Conf. Proc. 1015, 80 (2008), (arXiv:0801.3491 [hep-ph]).

[3] Y. Koide, Phys. Rev. D 78, 093006 (2008).

[4] Y. Koide, Phys. Lett. B 665, 227 (2008).

[5] P. F. Harrison, D. H. Perkins and W. G. Scott, Phys. Lett. B 458, 79 (1999); Phys. Lett. B 530, 167 (2002); Z.-z. Xing, Phys. Lett. B 533, 85 (2002); P. F. Harrison and W. G. Scott, Phys. Lett. B 535, 163 (2002); Phys. Lett. B 557, 76 (2003); E. Ma, Phys. Rev. Lett. 90, 221802 (2003); C. I. Low and R. R. Volkas, Phys. Rev. D 68, 033007 (2003).

[6] Y. Koide, arXive:0811.3470 [hep-ph].

[7] Y. Koide, Lett. Nuovo Cimento 34, 201 (1982); Phys. Lett. B 120, 161 (1983); Phys. Rev. D 28, 252 (1983).

[8] N. Li and B.-Q. Ma, Phys. Rev. D 73, 013009 (2008); X.-Z. Xing and H. Zhang, Phys. Lett. B 635,107 (2006); Y. Koide, J. Phys. G 34, 1653 (2007).

[9] Z.-z. Xing, H. Zhang and S. Zhou, Phys. Rev. D 77, 113016 (2008). Also, see H. Fusaoka and Y. Koide, Phys. Rev. D 57, 3986 (1998).

[10] Particle Data Group, Phys. Lett. B 667, 1 (2008).

[11] S. Abe, et al., KamLAND collaboration, Phys. Rev. Lett. 100, 221803 (2008).

[12] D. G. Michael et al., MINOS collaboration, Phys. Rev. Lett. 97, 191801 (2006); J. Hosaka, et al., Super-Kamiokande collaboration, Phys. Rev. D 74, 032002 (2006). 